U.S. Department of the Interior

U.S. Geological Survey

\title{
Location of Acoustic Sources Using Seismological Techniques and Software
}

by

J. C. Lahr and F. G. Fischer

Open-File Report 93-221

This report is preliminary and has not been reviewed for conformity with

U.S. Geological Survey editorial standards or with the North American Stratigraphic Code. Any use of trade, product, or firm names is for descriptive purposes only and does not imply endorsement by the U.S. Government.

Menlo Park, California

1993

Revised 3/31/93 


\section{Introduction}

During the summer of 1992, the Menlo Park Police Department received many reports of suspected gun shots in a neighborhood near the USGS. The police asked residents to phone 911 on these occasions, and on the basis of the geographic distribution of the callers, a police car was often sent to investigate. However, in most cases the police did not receive enough calls to accurately locate the source. Having each caller estimate the direction and distance of the sound was also of little use. Most callers reported shots heard while indoors, so the apparent direction was distorted by the acoustic parameters of the building, while the volume of the sound could not uniquely resolve both the distance to and magnitude of the source. Thus, it appeared that a more definitive approach to determining the source of a particular sound would be of benefit to the police.

\section{Similarity to the problem of locating earthquakes}

The problem of locating impulsive atmospheric acoustic sources is directly analogous to locating earthquakes. In both cases the times of first arrival of energy at an array of stations may be used to triangulate on the source of the energy. In one way earthquakes are easier to locate: for waves in the earth there are two types, $\mathrm{P}$ and $\mathrm{S}$, that travel with different speeds. The difference in arrival time at a given station between the faster $P$ wave and the slower $S$ wave is proportional to the distance the waves have travelled, so the $S$ wave arrival times provide very useful information to augment the $\mathrm{P}$ arrival times. On the other hand, both the geographic location (location on a map) and the depth must be determined for earthquakes. For an acoustic source there is just a $\mathrm{P}$ phase, so the slower $\mathrm{S}$ phase can not be used. However, if, as in this case, the sound sources and microphones are approximately at the same elevation, only the geographic location but not the elevation of the sound needs to be computed.

\section{Feasibility study}

It was decided to investigate the feasibility of using seismic techniques and software to locate acoustic sources. To do this a small array of five inexpensive dynamic microphones was deployed near the USGS. The dimensions of the array were $0.84 \mathrm{~km}$ by $0.28 \mathrm{~km}$. One microphone was located at the central recording site while the other four were radiotelemetered to the central site. Each microphone was mounted in a $4 \times 2 \times 1$ inch plastic box along with an amplifier. Various amplifier designs were tried and a schematic of one of these designs is shown in Figure 1. In this design the same batteries that powered the radio powered the amplifier. The remote microphone amplifiers were connected to the input of 100 miliwatt FM radios operating in the 160 to $170 \mathrm{Mhz}$ band using frequencies allocated to the USGS. Small log-periodic radio antennas were used at both the transmitter and the central receiver sites. 
At the central site, the four radio signals and the one local microphone amplifier were connected to an IBM compatible personal computer (PC) where they were digitized and continuously scanned for sudden increases in signal level. When a significant increase was detected at more than a set number of microphones, the software saved an "event" on the hard disk. The PC data acquisition, processing and analysis hardware are described by Lee and others (1989) and Tottingham and Lee (1989). The data acquisition software used in this study is derived from that of Lee and is described by Rogers (1993).

Once an event file was written to the hard disk, the arrival times of the energy at each station had to be determined. Although this process could be automated in the future, as will be discussed below, in this experiment the onsets were timed by hand using a version of the program PCEQ (Valdes, 1989) that was modified to allow a sampling rate higher than $100 \mathrm{~Hz}$ and to store arrival times to the nearest 0.001 second rather than 0.01 second as is common in seismology. Using PCEQ, the data from each microphone was graphically displayed, one microphone at a time. A mouse controlled cursor was used to time the onset of energy, which was recorded in a "pick" file. After all of the microphones with clear onsets were timed (a minimum of three arrival times are required by the mathematics) the program HYPOSHOT was run to locate the source.

HYPOSHOT uses the same iterative, damped least squares routines as the earthquake location program HYPOELLIPSE (Lahr, 1989). HYPOSHOT generates both a one-line summary file with the origin time and location of the sound as well as an information file which, in addition to the sound location, gives an estimate of the precision of the location and the residual at each microphone. The residual is the difference between the observed arrival time and the computed arrival time and should be small if the onset times have been correctly picked.

\section{Operational considerations}

Since all of the data were recorded in realtime at the central site, synchronization of the PC clock with some external standard, such as WWVB, was not required. The PC clock was maintained within about 1 minute of the time available from the phone company through dialing 767-1111.

After some experimentation with sampling rates and trigger thresholds, the seismic software proved to be quite effective at detecting and locating loud sounds. The sample rate was set at 2000 samples per second, which was high enough to capture signals with frequencies up to $1000 \mathrm{~Hz}$ and allow timing of the onsets to 0.001 second, but not so high as to become a major problem due to the size of the event files. At this sample rate, each 9 second event file is 144,144 bytes long.

The trigger algorithm is based on flags that can be raised at each station, based on the absolute values of the recent digital samples. If the ratio of the average of the previous 32 samples to the average of the previous 1024 samples exceeds a factor of 3.0 for 16 samples 
in a row, then the flag at that station goes up for 2 seconds. During the night, from about 7 $\mathrm{pm}$ to $7 \mathrm{am}$, if 3 or more flags were raised at the same time, an event with 9 seconds duration was saved. During the daytime, 4 or more flags were required to save an event. The threshold was raised during the daytime to avoid excessive false triggers. A false trigger might be caused by a hammer blow near one microphone within two seconds of a dog barking at another and a door slamming at a third. Due to the increased number of noise sources during the daytime, false triggers were much more common then than at night.

\section{Results}

During the time that all five microphones were in operation, many event files were saved which could have been gun shots. In the case of multiple shots from an automatic weapon, there is little doubt about their source (Figure 2). However, for an isolated event (Figure 3) some other impulsive source, such as a fire cracker or a motor vehicle backfire, also could have been the cause. A comparative study of the spectral and time domain characteristics of gun shots versus other impulsive sources might reveal differences that would be useful in discrimination.

Due to geometrical factors, the distance to sources that are outside of the array are poorly constrained while the azimuth to them is relatively well determined. Therefore, it would be best to deploy an array around the source region.

\section{Automation}

This study demonstrated the feasibility of using seismic data collection and analysis techniques to record and locate acoustic sources. As is practiced in seismology, a second PC can be connected to the on-line data collection PC via Lantastic brand network cards and software. A program on the second PC can monitor for recorded events and automatically locate events in near real time. Automation of this sort was beyond the scope of the current study, although a Lantastic network was used to manually retrieve data from the on-line computer, thus allowing it to run continuously. How well automated picking of the onsets of energy and subsequent location would work depends critically upon the microphone spacing. Impulsive sounds, such as gun shots, occurring within an array with microphone spacing of about 200 meters could probably be located automatically in most cases. However, other non-impulsive sounds would also be recorded by the current algorithm. This algorithm would have to be modified significantly to make it more reliable for use in a totally automated location system. However, if a graphical display of the microphone signals along with the automated picks were displayed, a person with little training could distinguish between a well located impulsive source and other noises. This might be the best compromise to use at the present time. 


\section{Alternate Approaches and Enhancements}

There are alternate approaches that could be used to locate the source of sounds. One suggested by $M$. Iyer would be to set up two or more small aperture arrays of microphones, each using beam-forming to locate the azimuth of the source. The signal-to-noise ratio would be enhanced by the beam-forming technique and fewer radio links would be required. In the current technique, correlation of the signals from each microphone might be useful in increasing the resolution of the relative arrival times, and thus improve location precision. For the cases of rapidly repeating stationary sources, stacking the signals at each microphone would also improve the signal-to-noise ratio and aid in timing.

\section{Acknowledgments}

Many people were helpful in setting up and completing this experiment and we especially thank John Coakley, Bob McClearn, John Rogers, Robert Banfill, Willie Lee, Mitch Robinson, and Janice Lahr. Mahadeva Iyer provided technical review of the manuscript. 


\section{References}

Lahr, J.C., 1989, HYPOELLIPSE/Version 2.0: A computer program for determining local earthquake hypocentral parameters, magnitude, and first motion pattern, U.S. Geological Survey Open-File Report 89-116, 92p.

Lee, W.H.K, Tottingham, D.M., and Ellis, J.O., 1989, Design and implementation of a PC-based seismic data acquisition, processing and analyiss system in: Lee, W.H.K.,editor, Toolbox for seismic data acquisition, processing and analysis, Published by International Association of Seismology and Physics of the Earth's Interior, P. 21-46.

Robinson, Mitch, 1990, XPICK user's manual v.2.7, Seismology Lab, Geophysical Institute, University of Alaska, 93p.

Rogers, J.A., 1993, XDETECT version 3.18 users reference guide, U.S. Geological Survey Open-File Report 93-xxx, 25p.

Tottingham, D.M. and Lee, W.H.K., 1989, User manual for XDETECT in: Lee, W.H.K., editor, Toolbox for seismic data acquisition, processing and analysis, Published by International Association of Seismology and Physics of the Earth's Interior, P. 89-118.

Valdes, C.M., 1989, User manual for PCEQ in: Lee, W.H.K., editor, Toolbox for seismic data acquisition, processing and analysis, Published by International Association of Seismology and Physics of the Earth's Interior, P. 175-201. 


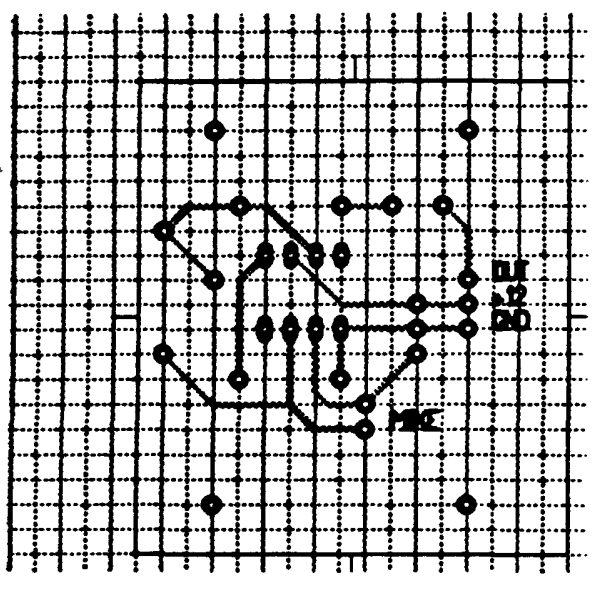

model 1
$250 \mu \mathrm{A}$ at $12 \mathrm{~V}$ supply $200 \mu \mathrm{A}$ at $6 \mathrm{Vsupply})$ wise code:

RED: + 12

WHITE: COM

GREEN: AUDIO OUT

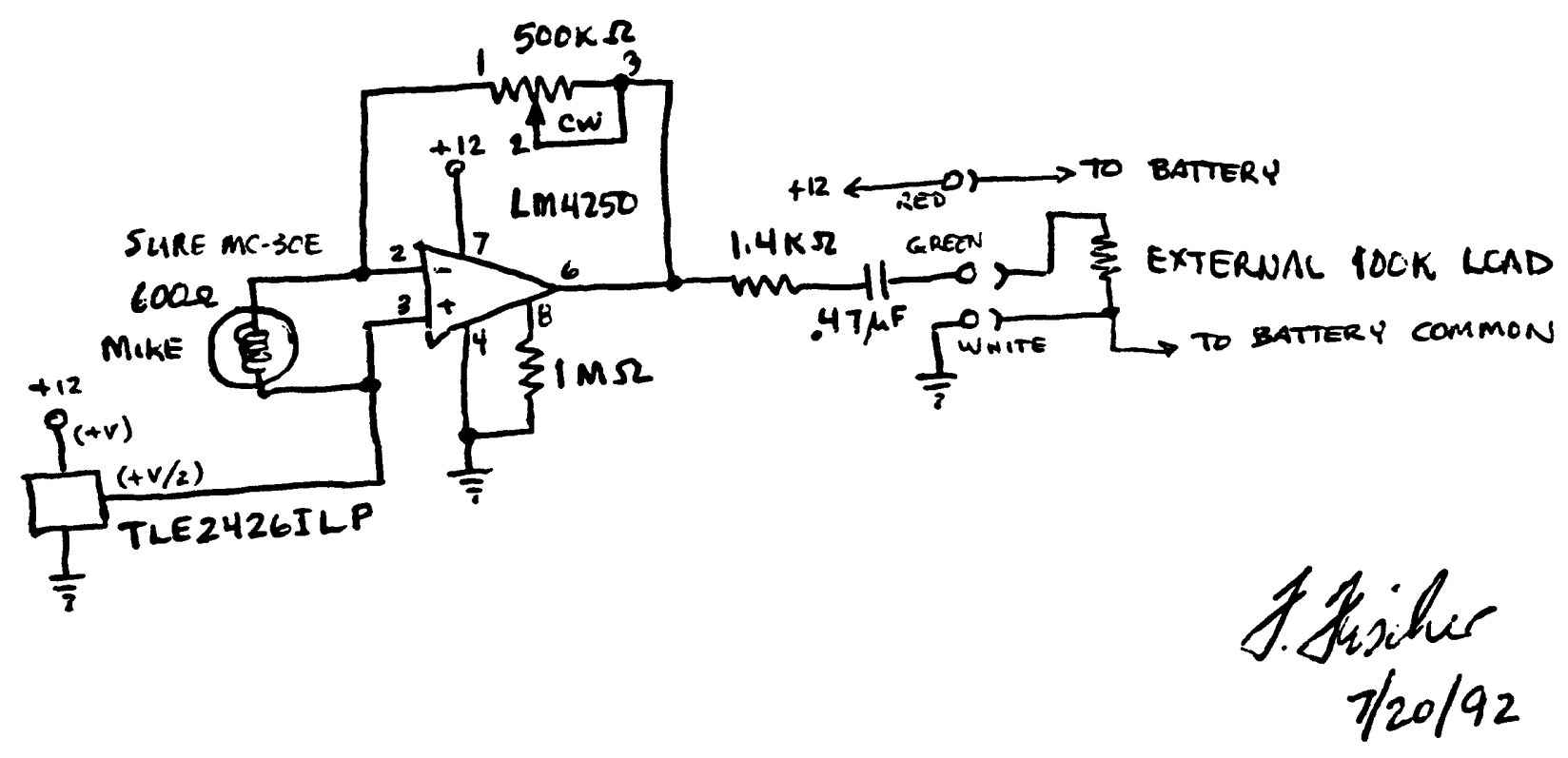

Figure 1. Schematic diagram of a typical microphone amplifier. 


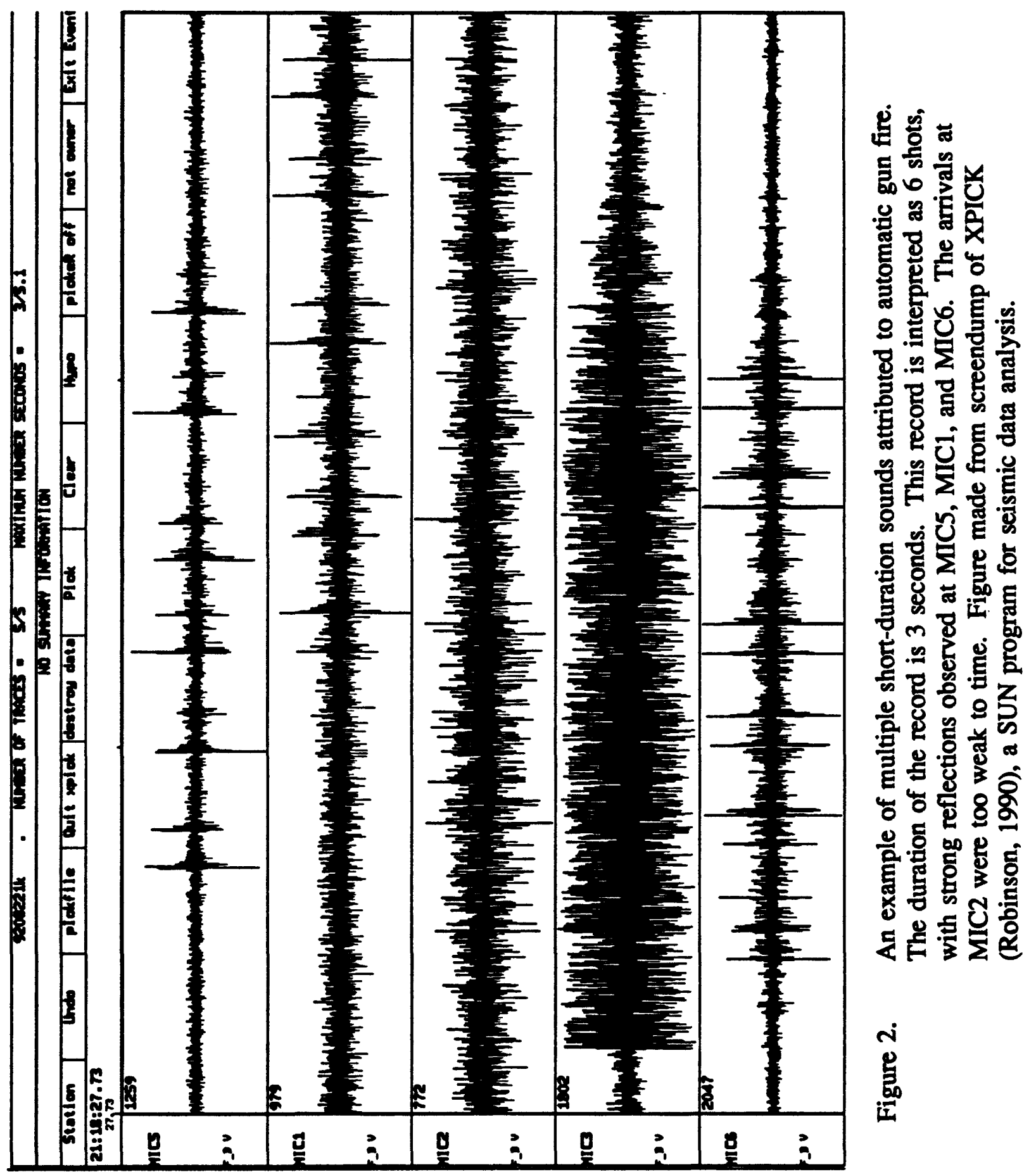




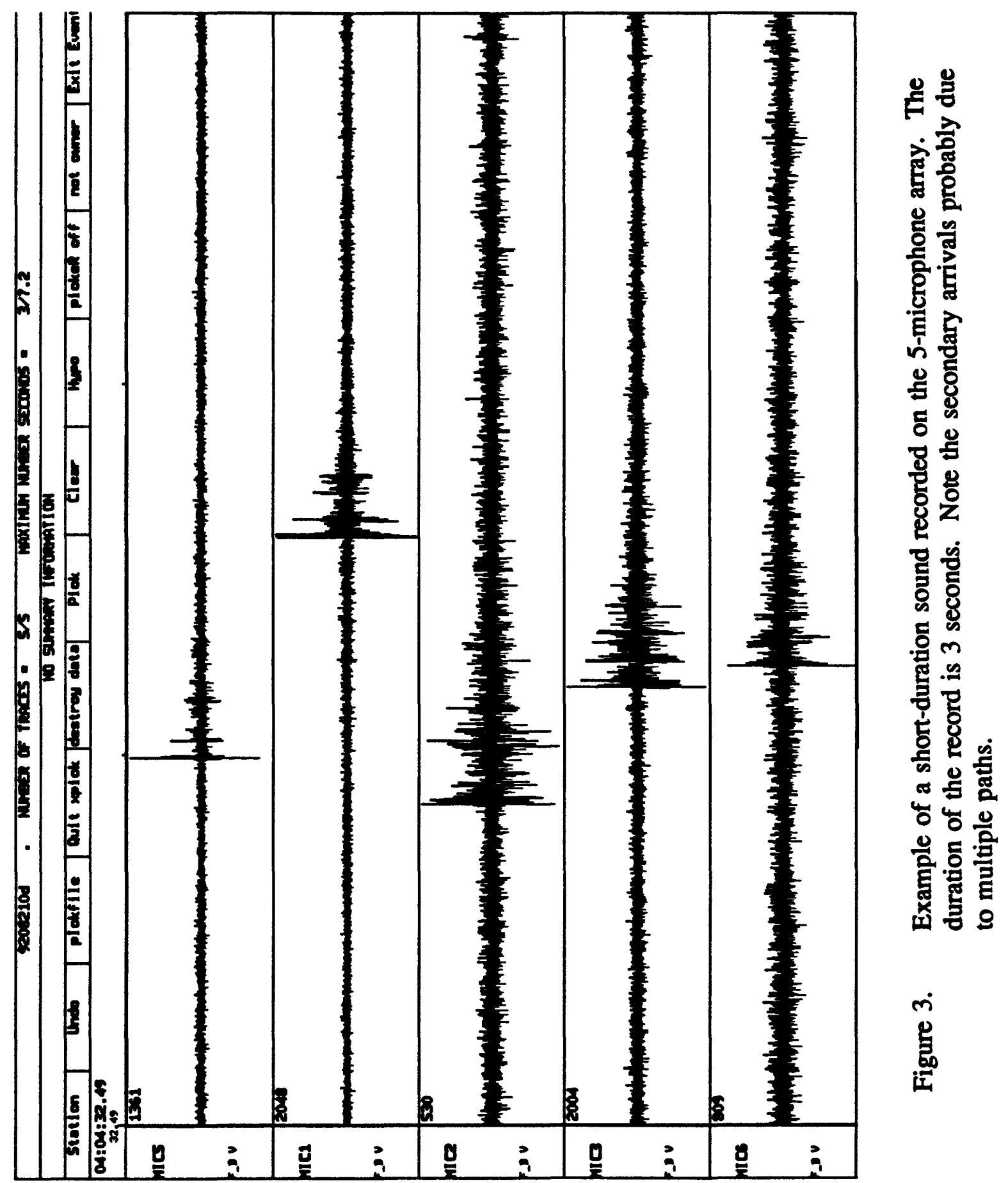

